Online -only supplemental material

\title{
Glutamine Regulates Skeletal Muscle Immunometabolism in Type 2 Diabetes
}

Lucile Dollet ${ }^{1}$, Michael Kuefner ${ }^{1}$, Elena Caria ${ }^{1}$, David Rizo-Roca ${ }^{2}$, Logan Pendergrast ${ }^{2}$, Ahmed M. Abdelmoez ${ }^{2}$, Håkan KR Karlsson ${ }^{2}$, Emilie Dalbram ${ }^{3}$, Jonas Treebak ${ }^{3}$, Jun Harada $^{4}$, Erik Näslund ${ }^{5}$, Mikael Rydén ${ }^{6}$, Juleen R. Zierath ${ }^{1,2}$, Nicolas J. Pillon ${ }^{1}$, Anna Krook ${ }^{1}$. ${ }^{1}$ Department of Physiology and Pharmacology, Integrative Physiology, Karolinska Institutet, 17177 Stockholm, Sweden. ${ }^{2}$ Department of Molecular Medicine and Surgery, Integrative, Physiology, Karolinska Institutet, 171 77, Stockholm, Sweden. ${ }^{3}$ Novo Nordisk Foundation Center for Basic Metabolic Research, Faculty of Health and Medical Sciences, University of Copenhagen, Copenhagen, Denmark. ${ }^{4}$ Cardiovascular-Metabolics Research Laboratories, Daiichi Sankyo Co., Ltd., Tokyo, Japan. ${ }^{5}$ Division of Surgery, Department of Clinical Sciences, Danderyd Hospital, Karolinska Institutet, 18288 Stockholm, Sweden. ${ }^{6}$ Department of Medicine, Huddinge, Karolinska Institutet Stockholm Sweden 


\begin{tabular}{|c|c|c|c|c|c|c|c|}
\hline Mouse gene & Ensembl reference & Human gene & Ensembl ref & mouse_logFC & mouse_P.Value & human_logFC & human_P.Value \\
\hline Defa22 & ENSMUSG00000074443 & DEFA5 & ENSG00000164816 & 0.092528 & 0.027769 & 0.140774 & 0.01513 \\
\hline Defa28 & ENSMUSG00000074434 & DEFA5 & ENSG00000164816 & -0.15117 & 0.016915 & 0.140774 & 0.01513 \\
\hline Defa40 & ENSMUSG00000074441 & DEFA5 & ENSG00000164816 & 0.084919 & 0.044609 & 0.140774 & 0.01513 \\
\hline Fnde1 & ENSMUSG00000071984 & FNDC1 & ENSG00000164694 & -0.17304 & 0.011134 & -0.16488 & 0.022891 \\
\hline Grb10 & ENSMUSG00000020176 & GRB10 & ENSG00000106070 & -0.14901 & 0.018904 & -0.15099 & 0.022287 \\
\hline Hba-a1 & ENSMUSG00000069919 & HBA1 & ENSG00000206172 & -1.03272 & 0.000178 & -1.13577 & 0.000698 \\
\hline Hba-a1 & ENSMUSG00000069919 & HBA2 & ENSG00000188536 & -1.03272 & 0.000178 & -1.27445 & 0.000917 \\
\hline Mrgprb2 & ENSMUSG00000050425 & MRGPRX4 & ENSG00000179817 & 0.112245 & 0.036967 & 0.217329 & 0.048118 \\
\hline Ptx4 & ENSMUSG00000044172 & PTX4 & ENSG00000251692 & -0.22896 & 0.000259 & -0.20994 & 0.018986 \\
\hline Rsad2 & ENSMUSG00000020641 & RSAD2 & ENSG00000134321 & -0.26204 & 0.00066 & -0.12742 & 0.02321 \\
\hline Snca & ENSMUSG00000025889 & SNCA & ENSG00000145335 & -0.18844 & 0.005769 & -0.17318 & 0.009612 \\
\hline Tas2r120 & ENSMUSG00000059382 & TAS2R31 & ENSG00000256436 & 0.087108 & 0.016376 & -0.19283 & 0.00415 \\
\hline Tas2r120 & ENSMUSG00000059382 & TAS2R19 & ENSG00000212124 & 0.087108 & 0.016376 & 0.32795 & 0.005263 \\
\hline Zfp992 & ENSMUSG00000070605 & ZNF34 & ENSG000000196378 & -0.08283 & 0.032177 & 0.10602 & 0.048793 \\
\hline
\end{tabular}

Supplemental Table 1: List of the genes commonly altered by glutamine administration in HFD fed mice $(\mathrm{p}<0.05)$ and altered in skeletal muscle of humans with high or low plasma glutamine levels $(\mathrm{p}<0.05)$. Log fold-change and adjusted $\mathrm{p}$-value are indicated for both mice and human dataset. 


\section{Supplemental Figure legends:}

Supplemental Figure 1: Details of the cohort recruitment and criteria for inclusion/exclusion (A). Volcano plots of metabolites in plasma (B) and skeletal muscle (C) correlating with waist/hip ratio (WHR). Spearman correlation and Wilcoxon comparison across groups. Blue color indicates significance at $\mathrm{p}<0.05$. Volcano plots of metabolites in plasma and skeletal muscle correlating with BMI and HOMA-IR (D-G). Spearman correlation and Wilcoxon comparison between NGT (lean+overweight) and T2D (lean+overweight) groups. Blue color indicates significance at $\mathrm{p}<0.05$ (D-G). Plasma glutamine and glutamate levels (H-I) and skeletal muscle glutamine and glutamate levels (J-K). Mann-Whitney test between NGT and T2D groups. 

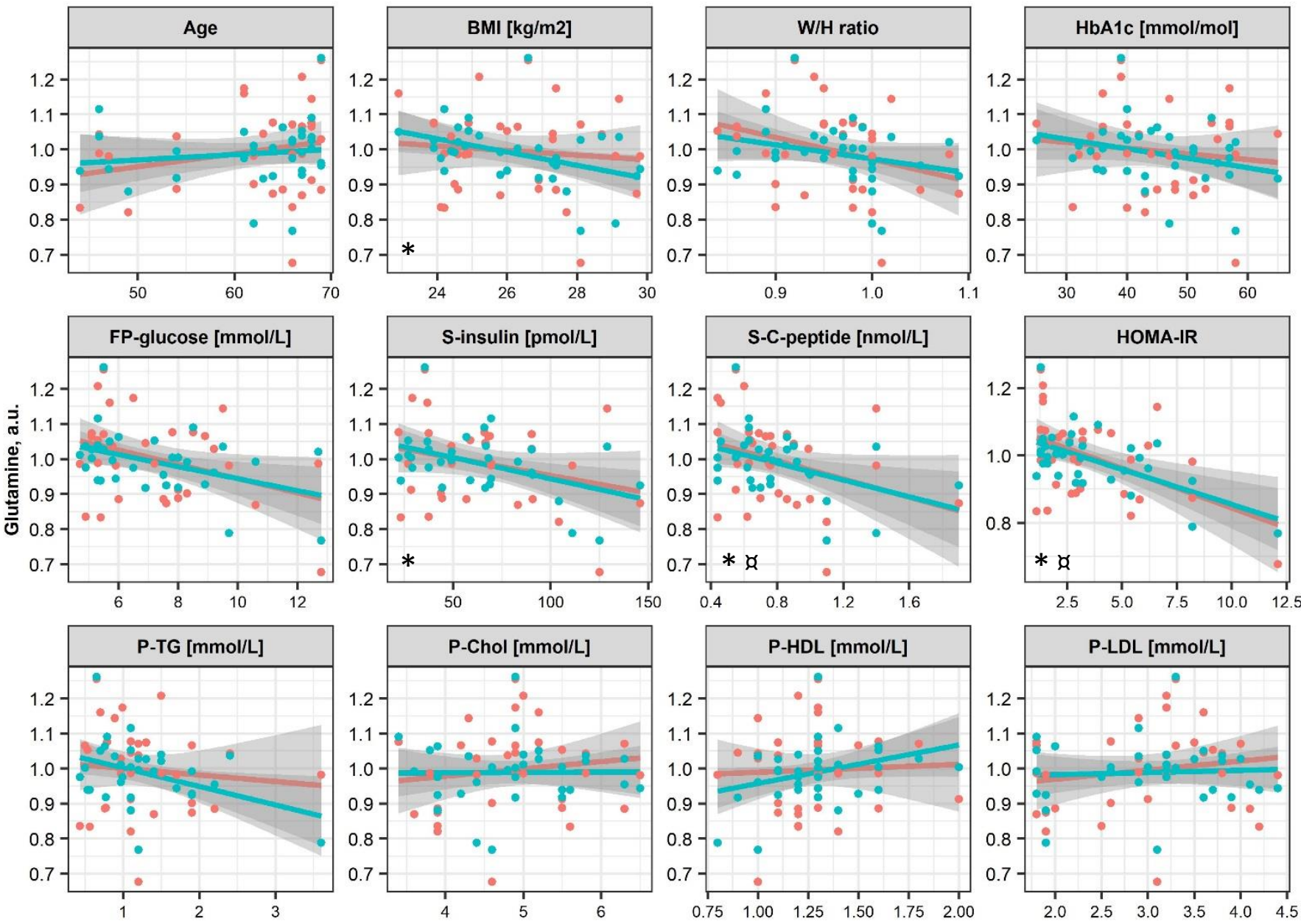

B
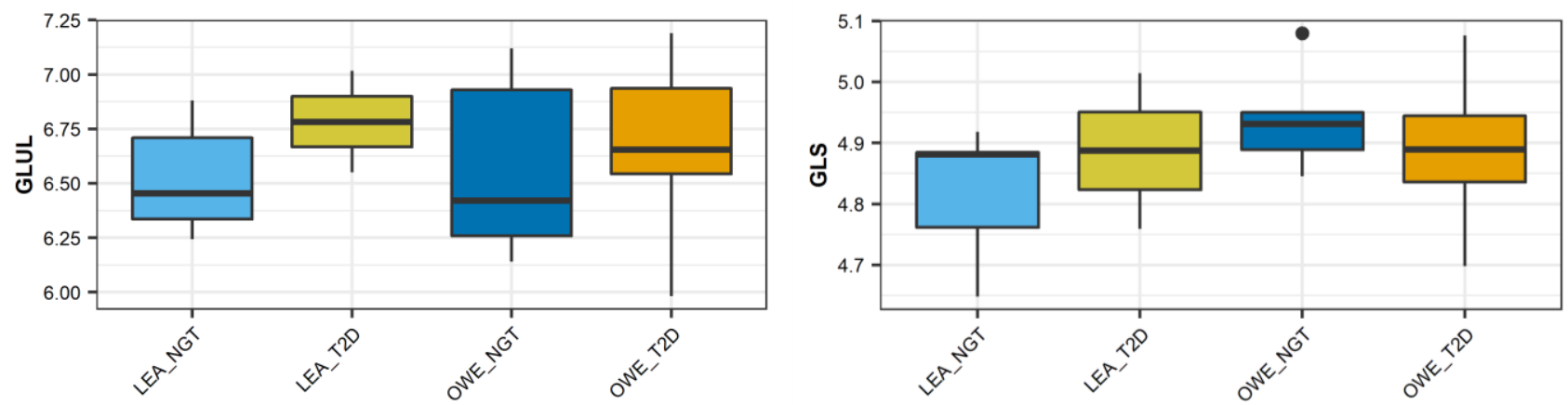
Supplemental Figure 2: Correlations between clinical parameters and plasma (blue) and muscle (red) glutamine level. *p $<0.05$ for plasma,, $0 \mathrm{p}<0.05$ for muscle (A). GLUL (B) and $G L S$ (C) gene expression level in human skeletal muscle. $n=27$. 

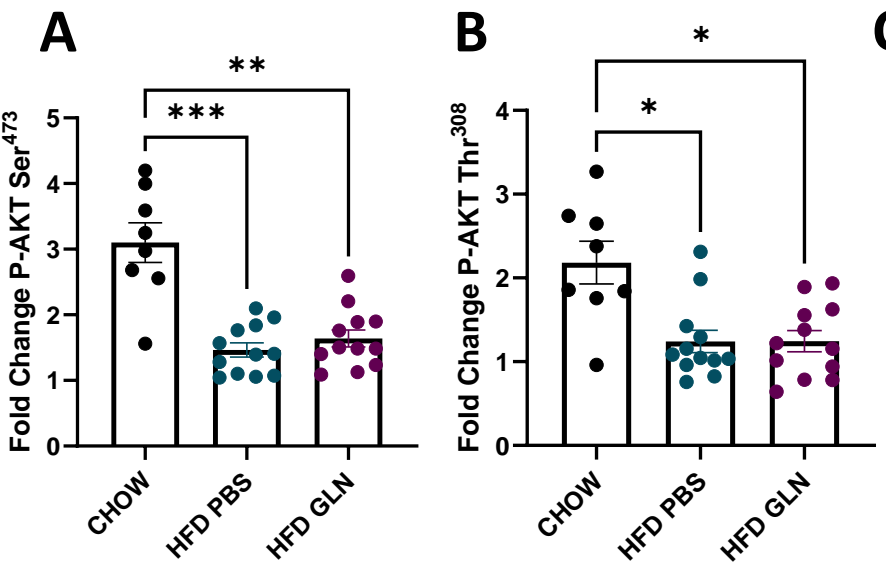

E
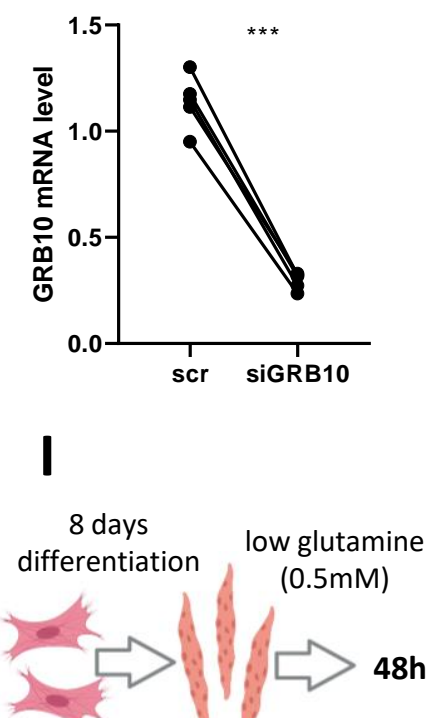

high glutamine

(10mM)

$\mathbf{F}$

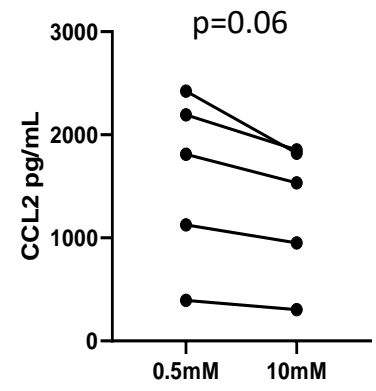

C

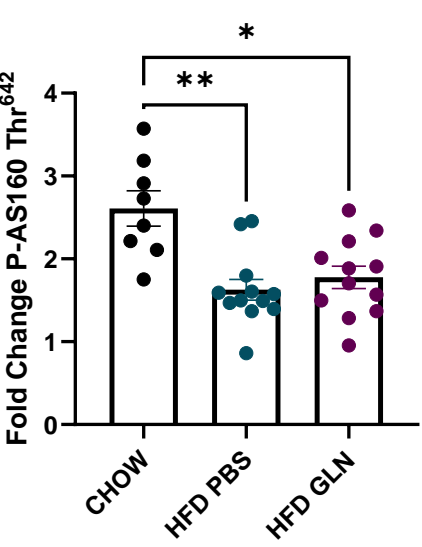

G
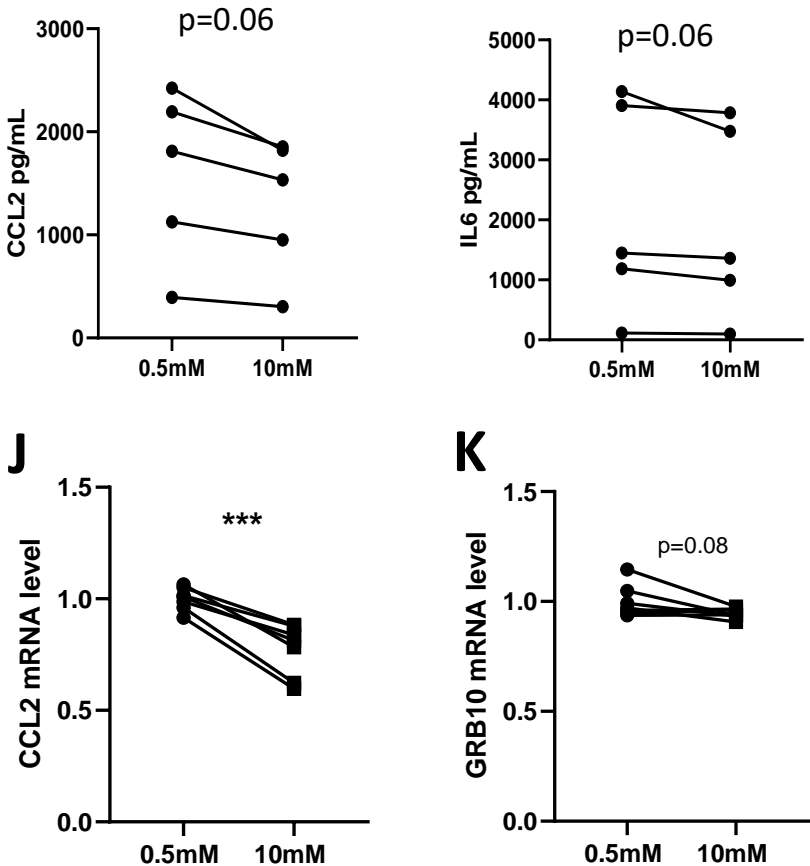

K

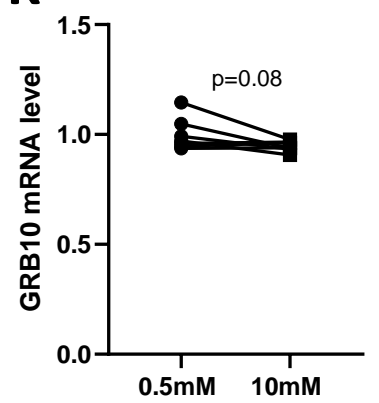

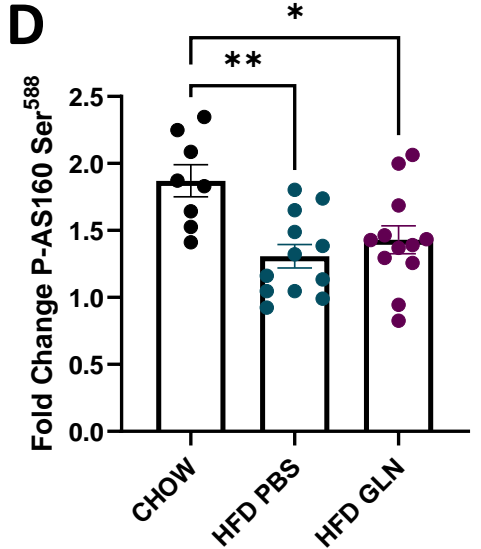

H

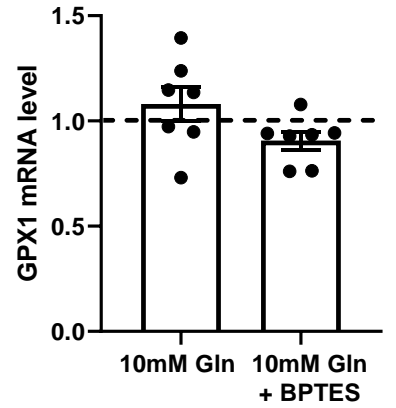

L

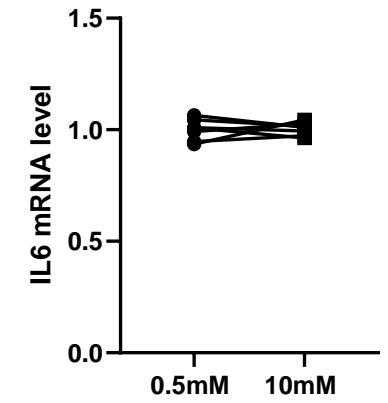




\section{Supplemental Figure 3:}

Fold-change in Akt phosphorylation (P-AKT) and AS160 phosphorylation (P-AS160) in lysates of soleus skeletal muscle incubated in the absence or presence of a submaximal dose of insulin $(0.36 \mathrm{nmol} / \mathrm{L})$ for $20 \mathrm{~min}(\mathrm{~A}-\mathrm{D})$. Data are mean \pm SEM. Kruskal-Wallis test with Dunn's multiple comparison test. GRB10 mRNA expression level in myotubes $48 \mathrm{~h}$ after transfection using either a scramble siRNA (scr) or a siRNA targeting GRB10 (siGRB10) (E). n=5. IL6 and CCL 2 content in the medium collected after $24 \mathrm{~h}$ from skeletal muscle cells differentiated during 8 days in low $(0.5 \mathrm{mM})$ or high $(10 \mathrm{mM})(\mathrm{F}-\mathrm{G}) . \mathrm{n}=5$. BPTES was added for $24 \mathrm{~h}$ and $G P X 1(\mathrm{H})$ mRNA level were measured. $\mathrm{n}=7$. Statistical effect was measured by paired Student's $t$-test. ${ }^{*} \mathrm{p}<0.05 ; * * \mathrm{p}<0.01 ; * * * \mathrm{p}<0.001$. Skeletal muscle cells were differentiated during 7 days in myotubes in classical $2 \mathrm{mM}$ glutamine medium, and then exposed to low $(0.5 \mathrm{mM})$ or high (10 $\mathrm{mM}$ ) concentration of glutamine during $48 \mathrm{~h}(\mathrm{I})$, and CCL2 (J), GRB10 (K) and IL6 (L) mRNA level were measured. $\mathrm{N}=7$. Statistical effect was measured by paired Student's $t$-test. ${ }^{*} \mathrm{p}<0.05$; $* * \mathrm{p}<0.01 ; * * * \mathrm{p}<0.001$ 